\title{
The 4-rank of the tame kernel versus the 4-rank of the narrow class group in quadratic number fields
}

by

Qin Yue (Shanghai and Jiangsu) and KeqIn Feng (Beijing)

1. Introduction. In the paper, we mainly investigate the relation between the 4-rank of the tame kernel of a quadratic number field $F=\mathbb{Q}(\sqrt{d})$ and the 4-rank of the narrow class group of a quadratic number field $E=$ $\mathbb{Q}(\sqrt{-d})$.

Let $O_{F}$ be the ring of integers of a number field $F$. For a finite Abelian group $A$, we shall denote by $A_{2}$ its 2 -Sylow subgroup, by ${ }_{2} A$ its subgroup consisting of elements of order at most 2 , by $r_{2}(A)$ its 2 -rank, and by $r_{4}(A)$ its 4-rank.

A large number of papers have contributed to determining the structure of the 2-Sylow subgroup of $K_{2} O_{F}$. By $[2,4,9]$ we have known 2-ranks and 4-ranks of $K_{2} O_{F}$ for general number fields $F$. Specifically, for quadratic fields $F$, J. Browkin and A. Schinzel [2] have given 2-rank formulas of $\mathrm{K}_{2} \mathrm{O}_{F}$, and H. Qin $[10,11]$ has got a method to calculate 4-ranks of $K_{2} O_{F}$. Recently, J. Hurrelbrink and M. Kolster [8] have generalized and improved the results of $[10,11]$ and have presented an effective way of computing 4-ranks of $\mathrm{K}_{2} \mathrm{O}_{F}$ for these relative quadratic extensions via the $F_{2}$-ranks of certain matrices (the analog of the Rédei matrices) of the local Hilbert symbol.

The aim of this paper is to show two formulas: for a real quadratic field $F=\mathbb{Q}(\sqrt{d})$ and an imaginary quadratic field $E=\mathbb{Q}(\sqrt{-d})$,

$$
r_{4}\left(K_{2} O_{F}\right)=a(F)+r_{4}(C(E)),
$$

where $C(E)$ is the narrow class group of $E$ and $a(F)=-1,0$, or 1 is determined by $F$;

$$
r_{4}\left(K_{2} O_{E}\right)=a(E)+r_{4}(C(F)),
$$

where $C(F)$ is the narrow class group of $F$ and $a(E)=-1,0$, or 1 is determined by $E$.

2000 Mathematics Subject Classification: 11R65, 11R70, 19C99, 19D50.

The paper is supported by the National Natural Science Foundation and Morningside Center of Math., CAS. 
We directly use the Rédei matrices to get the values of $a(F)$ and $a(E)$. On the other hand, for some imaginary quadratic fields, we give their Tate kernels.

2. Rédei's criteria. Let $F=\mathbb{Q}(\sqrt{d})$ be a quadratic field and $D$ the discriminant of $F$. We shall denote the narrow class group of $F$ by $C(F)$ and $N_{F / \mathbb{Q}}\left(F^{*}\right)$ by $N F$. Then

$$
r_{4}(C(F))=r_{2}\left({ }_{2} C(F) \cap C(F)^{2}\right) .
$$

L. Rédei [12] gave a criterion for $r_{4}(C(F))$. Let $D(F)$ be the set of all squarefree positive integers $q \mid D$. Then $D(F)$ is an elementary Abelian 2group with multiplication $q_{1} \cdot q_{2}=q_{1} q_{2} /\left(q_{1}, q_{2}\right)^{2}$. For $n(\neq 0) \in \mathbb{Z}$, we denote by $[n]$ the squarefree rational integer satisfying the relation $n=[n] a^{2}$ for some $a \in \mathbb{Z}$. Let $q \in D(F)$ and $q^{\prime}=[q D]$. We call $q$ a $D$-norm divisor if $q \in$ $N F$. Then $q$ is a $D$-norm divisor if and only if the equation $q x^{2}-q^{\prime} y^{2}-z^{2}=0$ has a non-trivial solution $x, y, z \in \mathbb{Z}$ if and only if $\left(\frac{q}{p}\right)=1$ for every odd prime $p \mid q^{\prime}$, and $\left(\frac{-q^{\prime}}{p}\right)=1$ for every odd prime $p \mid q$.

Let $D(N F)$ be the subgroup of $D(F)$ consisting of all $D$-norm divisors. For $q \in D(F)$, let $Q$ be the ideal of $F$ such that $(q)=Q^{2}$ and $\operatorname{cl}(Q) \in{ }_{2} C(F)$ be the narrow ideal class containing $Q$. Rédei proved that $\operatorname{cl}(Q) \in C(F)^{2}$ if and only if $q \in D(N F)$ by the Gauss theorem and that the map

$$
\alpha: D(N F) \rightarrow{ }_{2} C(F) \cap C(F)^{2}, \quad q \mapsto \operatorname{cl}(Q),
$$

is a surjective homomorphism with $|\operatorname{ker} \alpha|=2$. Hence

$$
r_{4}(C(F))=r_{2}(D(N F))-1 .
$$

In particular, if $D<0$, then $\operatorname{ker} \alpha=\{1,[-D]\}$, and we have $q \in D(N F)$ if and only if $-q^{\prime} \in D(N F)$.

Rédei also related a criterion for $r_{4}(C(F))$ to the rank of a certain matrix with coefficients in $\mathbb{Z} / 2 \mathbb{Z}$. Suppose that a positive integer $n$ is prime to $D$; we shall write $a=\left(\frac{D}{n}\right)^{\prime}$ if the Jacobi symbol $\left(\frac{D}{n}\right)=(-1)^{a}$ with $a \in \mathbb{Z} / 2 \mathbb{Z}$. The discriminants $p^{*}=(-1)^{(p-1) / 2} p$ ( $p$ odd prime), $-4,8,-8(p=2)$ are called prime discriminants. Let $D=p_{1}^{*} \ldots p_{t}^{*}$ be the unique decomposition of $D$ into a product of prime discriminants. In the case $2 \mid D$, put $p_{t}=2$. We define a $t \times t$ square matrix $A_{F}=\left(a_{i j}\right)$ with coefficients in $\mathbb{Z} / 2 \mathbb{Z}$ by

$$
a_{i j}= \begin{cases}\left(\frac{p_{i}^{*}}{p_{j}}\right)^{\prime} & \text { if } i \neq j, \\ \left(\frac{D / p_{i}^{*}}{p_{i}}\right)^{\prime} & \text { if } i=j .\end{cases}
$$

Note that the sum of all rows of $A_{F}$ is 0 . 
Let $A_{F}^{\prime}$ be the $(t-1) \times t$ matrix obtained from $A_{F}$ by deleting the $t$ th row. Then $\operatorname{rank} A_{F}^{\prime}=\operatorname{rank} A_{F}$. By the reciprocity law, we have

$$
\begin{aligned}
A_{F}^{\prime} & =\left(\begin{array}{ccccc}
\left(\frac{D / p_{1}^{*}}{p_{1}}\right)^{\prime} & \left(\frac{p_{1}^{*}}{p_{2}}\right)^{\prime} & \ldots & \left(\frac{p_{1}^{*}}{p_{t-1}}\right)^{\prime} & \left(\frac{p_{1}^{*}}{p_{t}^{*}}\right)^{\prime} \\
\left(\frac{p_{t-1}^{*}}{p_{1}}\right)^{\prime} & \left(\frac{p_{t-1}^{*}}{p_{2}}\right)^{\prime} & \ldots & \left(\frac{D / p_{t-1}^{*}}{p_{t-1}^{*}}\right)^{\prime} & \left(\frac{p_{t-1}^{*}}{p_{t}}\right)^{\prime}
\end{array}\right) \\
& =\left(\begin{array}{ccccc}
\left(\frac{D / p_{1}^{*}}{p_{1}}\right)^{\prime} & \left(\frac{p_{2}}{p_{1}}\right)^{\prime} & \ldots & \left(\frac{p_{t-1}}{p_{1}}\right)^{\prime} & \left(\frac{p_{t}}{p_{1}}\right)^{\prime} \\
\cdots & \cdots & \ldots & \widetilde{D} & \cdots \\
\left(\frac{p_{1}}{p_{t-1}}\right)^{\prime} & \left(\frac{p_{2}}{p_{t-1}}\right)^{\prime} & \ldots & \left(\frac{D / p_{t-1}^{*}}{p_{t-1}}\right)^{\prime} & \left(\frac{p_{t}}{p_{t-1}}\right)^{\prime}
\end{array}\right) .
\end{aligned}
$$

For $q \in D(F)$, we define $X_{q}={ }^{t}\left(x_{1}, \ldots, x_{t}\right) \in(\mathbb{Z} / 2 \mathbb{Z})^{t}$ by

$$
x_{i}=\left\{\begin{array}{ll}
1 & \left(p_{i} \mid q\right) \\
0 & \left(p_{i} \nmid q\right)
\end{array} \quad(i=1, \ldots, t) .\right.
$$

Then we have $A_{F}^{\prime} X_{q}=0$ if and only if $A_{F} X_{q}=0$ if and only if

$$
\begin{cases}\left(\frac{q}{p}\right)=1 & \text { for every odd prime } p \mid q^{\prime} \\ \left(\frac{(q / p)\left(D / p^{*}\right)}{p}\right)=1 & \text { for every odd prime } p \mid q,\end{cases}
$$

if and only if $\left(\frac{q}{p}\right)=1$ for every odd prime $p \mid q^{\prime}$, and $\left(\frac{-q^{\prime}}{p}\right)=1$ for every odd prime $p \mid q$, if and only if the equation $q x^{2}-q^{\prime} y^{2}-z^{2}=0$ has a non-trivial solution $x, y, z \in \mathbb{Z}$. Hence, the map

$$
\theta: D(N F) \rightarrow\left\{y \in(\mathbb{Z} / 2 \mathbb{Z})^{t} \mid A_{F} X_{q}=0\right\}, \quad q \mapsto X_{q},
$$

is an isomorphism, and we have

$$
r_{4}(C(F))=r_{2}(D(N F))-1=t-1-\operatorname{rank} A_{F} .
$$

3. Real quadratic fields. In the section, let $F=\mathbb{Q}(\sqrt{d})$ be a real quadratic field, and $d>2$ a squarefree integer. J. Browkin and A. Schinzel [2] have given all elements of order 2 of $\mathrm{K}_{2} \mathrm{O}_{F}$.

Lemma 3.1. Let $F=\mathbb{Q}(\sqrt{d}), d>2$ a squarefree integer, and $p$ a fixed odd prime divisor of $d$. Then all elements of order at most 2 of $\mathrm{K}_{2} \mathrm{O}_{F}$ are of the form

$$
\left\{-1, m \gamma_{j}\right\},
$$

where $m$ is an odd divisor of $d$ positive and negative but $p \nmid m, \gamma_{1}=1$, and $\gamma_{j}=u_{j}+\sqrt{d}, u_{j}^{2}-j w_{j}^{2}=d, u_{j}, w_{j} \in \mathbb{N}, j \in\{-1, \pm 2\} \cap N F$.

In [10], H. Qin has given conditions for $K_{2} O_{F}$ to have elements of order 4 .

Lemma 3.2. Let $F=\mathbb{Q}(\sqrt{d}), d>2$ a squarefree integer, and $m$ an odd positive divisor of $d$. 
(1) There is a $\beta \in K_{2} O_{F}$ such that $\beta^{2}=\{-1, m\}$ if and only if there is an $\varepsilon \in\{ \pm 1, \pm 2\}$ such that

$$
\begin{aligned}
\left(\frac{\varepsilon d m^{-1}}{p}\right) & =1 & & \text { for every odd prime } p \mid m, \\
\left(\frac{\varepsilon m}{p}\right) & =1 & & \text { for every odd prime } p \mid d m^{-1} .
\end{aligned}
$$

(2) If $2 \in N F, d=u^{2}-2 w^{2}, u, w \in \mathbb{N}$, then there is a $\beta \in K_{2} O_{F}$ such that $\beta^{2}=\{-1, m(u+\sqrt{d})\}$ if and only if there is an $\varepsilon \in\{ \pm 1\}$ such that

$$
\begin{array}{rlrl}
\left(\frac{\varepsilon d m^{-1}(u+w)}{p}\right) & =1 & & \text { for every odd prime } p \mid m, \\
\left(\frac{\varepsilon m(u+w)}{p}\right) & =1 & \text { for every odd prime } p \mid d m^{-1} .
\end{array}
$$

In what follows, we shall investigate the conditions (3.1) and (3.2) to set up the relation between the 4-rank of $K_{2} O_{F}$ of the real quadratic field $F=\mathbb{Q}(\sqrt{d})$ and the 4-rank of the narrow class group $C(E)$ of the imaginary field $E=\mathbb{Q}(\sqrt{-d})$.

Definition 3.1. Let $F=\mathbb{Q}(\sqrt{d}), d>2$ a squarefree integer. We define

$S_{0}=\{m \mid m$ is an odd positive divisor of $d\}$,

$S_{1}=\left\{\varepsilon m \mid m \in S_{0}\right.$ and $\varepsilon \in\{1,2\}$ satisfy $\left.(3.1)\right\}$,

$S_{2}=\left\{|\varepsilon| m \mid m \in S_{0}\right.$ and $\varepsilon \in\{-1,-2\}$ satisfy (3.1), but $\left.m, 2 m \notin S_{1}\right\}$.

If $2 \in N F, d=u^{2}-2 w^{2}, u, w \in \mathbb{N}$, we define

$$
\begin{aligned}
& S_{1}^{\prime}=\left\{m(u+\sqrt{d}) \mid m \in S_{0} \text { and } \varepsilon=1 \text { satisfy }(3.2)\right\}, \\
& S_{2}^{\prime}=\left\{m(u+\sqrt{d}) \mid m \in S_{0} \text { and } \varepsilon=-1 \text { satisfy }(3.2), \text { but } m \notin S_{1}^{\prime}\right\} .
\end{aligned}
$$

In fact, if -1 or -2 is in $N F$, then $S_{2}=S_{2}^{\prime}=\emptyset$. Similarly to $D(F)$, we define $\bar{S}_{1}=S_{1}$, which is an elementary Abelian 2-group, and $\bar{S}_{2}=$ $\left(S_{2} \cup S_{1}\right)$ is the group generated by the set $S_{2} \cup S_{1}$ with multiplication $m_{1} \cdot m_{2}=m_{1} m_{2} /\left(m_{1}, m_{2}\right)^{2}$. If $2 \in N F, u^{2}-2 w^{2}=d, u, w \in \mathbb{N}$, we define $\bar{S}_{1}^{\prime}=\left(S_{1}^{\prime} \cup S_{1}\right)$ to be the group generated by the set $S_{1}^{\prime} \cup S_{1}$ and $\bar{S}_{2}^{\prime}=$ $\left(S_{2}^{\prime} \cup S_{1}\right)$ to be the group generated by the set $S_{2}^{\prime} \cup S_{1}$ with multiplication $\left(m_{1}(u+\sqrt{d})\right) \cdot m_{2}=\left(m_{1} \cdot m_{2}\right)(u+\sqrt{d}),\left(m_{1}(u+\sqrt{d})\right) \cdot\left(m_{2}(u+\sqrt{d})\right)=m_{1} \cdot m_{2}$.

LEMMA 3.3. Notations as above.

(1) If $2 \notin N F$, then $r_{4}\left(K_{2} O_{F}\right)=r_{2}\left(S_{1}\right)+s-1$, where

$$
s= \begin{cases}1 & \text { if } S_{2} \neq \emptyset, \\ 0 & \text { if } S_{2}=\emptyset .\end{cases}
$$


(2) If $2 \in N F$, then $r_{4}\left(K_{2} O_{F}\right)=r_{2}\left(S_{1}\right)+s^{\prime}-2$, where

$$
s^{\prime}= \begin{cases}2 & \text { if } S_{2}, S_{1}, S_{2}^{\prime} \text { are all non-empty, } \\ 1 & \text { if only one of } S_{2}, S_{1}^{\prime}, S_{2}^{\prime} \text { is non-empty } \\ 0 & \text { if } S_{2}=S_{1}^{\prime}=S_{2}^{\prime}=\emptyset .\end{cases}
$$

Proof. (1) Let $2 \notin N F$. Suppose $S_{2} \neq \emptyset$, so take $m \in S_{2}$. Then $m S_{1}=$ $\left\{m \cdot m_{1} \mid m_{1} \in S_{1}\right\}=S_{2}$ and $m S_{2}=\left\{m \cdot m_{2} \mid m_{2} \in S_{2}\right\}=S_{1}$. Hence $S=\bar{S}_{1} \bar{S}_{2}=(m) \times S_{1}$. By Lemmas 3.1 and 3.2, the map $\gamma: S \rightarrow{ }_{2} K_{2} O_{F} \cap$ $\left(K_{2} O_{F}\right)^{2}, a \mapsto\{-1, a\}$, is a surjective homomorphism of two groups, and $\operatorname{ker} \gamma=(d) \subset S_{1}$. Therefore $r_{4}\left(K_{2} O_{F}\right)=r_{2}\left({ }_{2} K_{2} O_{F} \cap\left(K_{2} O_{F}\right)^{2}\right)=r_{2}(S)-1$ $=r_{2}\left(S_{1}\right)-1+s$, where $s=0$ if $S_{2}^{\prime}=\emptyset$ or $s=1$ if $S_{2}^{\prime} \neq \emptyset$.

(2) Let $2 \in N F$. Similarly, if $S_{i}^{\prime} \neq \emptyset$, then $\bar{S}_{i}^{\prime}=(m(u+\sqrt{d})) \times S_{1}$, where $m(u+\sqrt{d}) \in S_{i}^{\prime}, i=1,2$; if two of $S_{2}, S_{1}^{\prime}, S_{2}^{\prime}$ are non-empty, then the third is non-empty; if $S_{2}, S_{1}^{\prime}, S_{2}^{\prime}$ are all non-empty, then $S=\bar{S}_{1} \bar{S}_{2} \bar{S}_{1}^{\prime} \bar{S}_{2}^{\prime}=$ $(m) \times\left(m_{1}(u+\sqrt{d})\right) \times S_{1}$, where $m \in S_{2}$ and $m_{1}(u+\sqrt{d}) \in S_{1}^{\prime}$. On the other hand, the map $\gamma^{\prime}: S \rightarrow{ }_{2} K_{2} O_{F} \cap\left(K_{2} O_{F}\right)^{2}, a \mapsto\{-1, a\}$, is a surjective homomorphism and ker $\gamma^{\prime}=(2) \times(d) \subset S_{1}$. Hence $r_{4}\left(K_{2} O_{F}\right)=r_{2}\left({ }_{2} K_{2} O_{F} \cap\right.$ $\left.\left(K_{2} O_{F}\right)^{2}\right)=r_{2}(S)-2=r_{2}\left(S_{1}\right)+s^{\prime}-2$, where $s^{\prime}=0$ if $S_{2}=S_{1}^{\prime}=S_{2}^{\prime}=\emptyset$, or $s^{\prime}=1$ if only one of $S_{2}, S_{1}^{\prime}, S_{2}^{\prime}$ is non-empty, or $s^{\prime}=2$ if $S_{2}, S_{1}^{\prime}, S_{2}^{\prime}$ are all non-empty.

Lemma 3.4. Notations as above. Suppose $d \equiv-1 \bmod 8$. Then $S_{2}=\emptyset$ and $S_{2}^{\prime}=\emptyset$ if $2 \in N F$.

Pr o of. Suppose odd $m \in S_{2}$. Then $\left(\frac{d m^{-1}}{p}\right)=\left(\frac{-1}{p}\right)$ for every odd prime $p \mid m$, and $\left(\frac{m}{p}\right)=\left(\frac{-1}{p}\right)$ for every odd prime $p \mid d m^{-1}$. By $d \equiv-1 \bmod 8$ and the quadratic reciprocity law, $\left(\frac{d m^{-1}}{m}\right)=\left(\frac{m}{d m^{-1}}\right)$, so $\left(\frac{-1}{m}\right)=\left(\frac{-1}{d m^{-1}}\right)$, which is contradictory. Similarly, we can prove that there is no even $2 m \in S_{2}$.

Let $2 \in N F, u^{2}-2 w^{2}=d, u, w \in \mathbb{N}$. Suppose $m(u+\sqrt{d}) \in S_{2}^{\prime}$. Then $\left(\frac{m(u+w)}{p}\right)=\left(\frac{-1}{p}\right)$ for every odd prime $p \mid d m^{-1}$ and $\left(\frac{d m^{-1}(u+w)}{p}\right)=\left(\frac{-1}{p}\right)$ for every odd prime $p \mid m$. By $d \equiv-1 \bmod 8$ and the quadratic reciprocity law, $\left(\frac{d m^{-1}}{m}\right)=\left(\frac{m}{d m^{-1}}\right)$. Also $2(u+w)^{2}=d+(u+2 w)^{2}$ and let $u+w=2^{i}(\overline{u+w})$, where $\overline{u+w}$ is odd. Then $1=\left(\frac{-d}{\overline{u+w}}\right)=\left(\frac{-m d m^{-1}}{\overline{u+w}}\right)$. Hence $\left(\frac{\overline{u+w}}{d m^{-1}}\right)=\left(\frac{\overline{u+w}}{m}\right)$ by $d \equiv-1 \bmod 8$ and the quadratic reciprocity. Therefore $\left(\frac{-1}{m}\right)=\left(\frac{-1}{d m^{-1}}\right)$, contrary to $d \equiv-1 \bmod 8$.

It is clear that $S_{1}$ is related to the group $D(N E)$ of the quadratic field $E=\mathbb{Q}(\sqrt{-d})$, which is defined as in the second section, so we can get the following formula.

Theorem 3.1. Let $F=\mathbb{Q}(\sqrt{d}), E=\mathbb{Q}(\sqrt{-d}), d>2$ a squarefree integer, and $C(E)$ the (narrow) class group of $E$. 
(1) If $2 \notin N F$, then $r_{4}\left(K_{2} O_{F}\right)=r_{4}(C(E))+s$, where

$$
s= \begin{cases}1 & \text { if } S_{2} \neq \emptyset, \text { or } d \equiv-1 \bmod 8 \text { and even } 2 m \in S_{1}, \\ 0 & \text { otherwise. }\end{cases}
$$

(2) If $2 \in N F$, then

$$
r_{4}\left(K_{2} O_{F}\right)= \begin{cases}r_{4}(C(E))+s^{\prime}-1 & \text { if } d \not \equiv-1 \bmod 8 \\ r_{4}(C(E))+s^{\prime} & \text { if } d \equiv-1 \bmod 8\end{cases}
$$

where

$$
s^{\prime}= \begin{cases}2 & \text { if } S_{2}, S_{1}, S_{2}^{\prime} \text { are all empty } \\ 1 & \text { if only one of } S_{2}, S_{1}^{\prime}, S_{2}^{\prime} \text { is non-empty } \\ 0 & \text { if } S_{2}=S_{1}^{\prime}=S_{2}^{\prime}=\emptyset\end{cases}
$$

Moreover, $r_{4}\left(K_{2} O_{F}\right)=r_{4}(C(E))+a(F)$, where $a(F)=-1,0$, or 1 is determined by $F$.

Pr o of. By Lemmas 3.3 and 3.4, it is sufficient to find the relation between $r_{2}\left(S_{1}\right)$ and $r_{4}(C(E))$.

(1) Let $2 \notin N F$. Suppose $d \not \equiv-1 \bmod 4$. Then $2 \mid D$, where $D$ is the discriminant of $E=\mathbb{Q}(\sqrt{-d})$, so $D(N E)=S_{1}$. Hence $r_{4}(C(E))=$ $r_{2}(D(N F))-1=r_{2}\left(S_{1}\right)-1$.

Suppose $d \equiv-5 \bmod 8$. Then $2 \nmid D$. Also there is no even $2 m \in S_{1}$ by the quadratic reciprocity law (or $\left(\frac{2 d m^{-1}}{m}\right)=\left(\frac{2 m}{d m^{-1}}\right)$, which is contradictory). Hence $D(N E)=S_{1}$, so $r_{4}(C(E))=r_{2}\left(S_{1}\right)-1$.

Suppose that $d \equiv-1 \bmod 8$ and there is an even $2 m \in S_{1}$. Then $S_{1}=$ $(2 m) \times D(N E)$, so $r_{4}(C(E))=r_{2}\left(S_{1}\right)-2$.

(2) If $2 \in N F$, then $2 \in S_{1}$. Suppose $d \not \equiv-1 \bmod 4$. Then $2 \mid D$, where $D$ is the discriminant of $E$, and $D(N E)=S_{1}$, so $r_{4}(C(E))=r_{2}\left(S_{1}\right)-1$. Suppose $d \equiv-1 \bmod 8$. Then $2 \nmid D(=-d)$ and $S_{1}=(2) \times D(N E)$, so $r_{4}(C(E))=r_{2}\left(S_{1}\right)-2$.

In Theorem 3.1, in order to get the value of $r_{4}\left(K_{2} O_{F}\right)$ clearly, we use the Rédei matrix to determine if $S_{2}, S_{1}^{\prime}, S_{2}^{\prime}$ are empty.

TheOREM 3.2. Let $F=\mathbb{Q}(\sqrt{d}), E=\mathbb{Q}(\sqrt{-d})$, and $d>2$ a squarefree integer.

(1) If $2 \notin N F$ and $d \equiv-1 \bmod 8$, then there is an even $2 m \in S_{1}$ if and only if the system of equations

$$
A_{E}^{\prime} X=B^{\prime}
$$

is solvable, where $B^{\prime}={ }^{t}\left(\left(\frac{2}{p_{1}}\right)^{\prime}, \ldots,\left(\frac{2}{p_{t-1}}\right)^{\prime}\right)$ and $A_{E}^{\prime}$ is defined as (2.2).

(2) If $-1,-2 \notin N F$, then $S_{2}=\emptyset$ if and only if the system (3.3) has no solution, where $B^{\prime}={ }^{t}\left(\left(\frac{-1}{p_{1}}\right)^{\prime}, \ldots,\left(\frac{-1}{p_{t-1}}\right)^{\prime}\right)$ if $d \not \equiv-1 \bmod 4$ and $B^{\prime}=$ ${ }^{t}\left(\left(\frac{-2}{p_{1}}\right)^{\prime}, \ldots,\left(\frac{-2}{p_{t-1}}\right)^{\prime}\right)$ if $d \equiv 3 \bmod 8$. 
(3) If $2 \in N F$, then $S_{1}^{\prime}=\emptyset$ if and only if the system (3.3) has no solution, where $B^{\prime}={ }^{t}\left(\left(\frac{u+w}{p_{1}}\right)^{\prime}, \ldots,\left(\frac{u+w}{p_{t-1}}\right)^{\prime}\right)$.

(4) If $2 \in N F,-1 \notin N F$, and $d \not \equiv-1 \bmod 8$, then $S_{2}^{\prime}=\emptyset$ if and only if the system (3.3) has no solution, where $B^{\prime}={ }^{t}\left(\left(\frac{-u-w}{p_{1}}\right)^{\prime}, \ldots,\left(\frac{-u-w}{p_{t-1}}\right)^{\prime}\right)$.

Proof. (1) If $d \equiv-1 \bmod 8$ and $2 \notin N F$, then $D=-d$ is the discriminant of $E$ and $1=\left(\frac{2}{d}\right)=\left(\frac{2}{p_{1}}\right) \ldots\left(\frac{2}{p_{t}}\right)$. For $2 m \in S_{1}$, we define $X_{m}={ }^{t}\left(x_{1}, \ldots, x_{t}\right) \in(\mathbb{Z} / 2 \mathbb{Z})^{t}$ by

$$
x_{i}= \begin{cases}1 & \text { if } p_{i} \mid m, \\ 0 & \text { if } p_{i} \nmid m,\end{cases}
$$

where $i=1, \ldots, t$. So we have $A_{E}^{\prime} X_{m}=B^{\prime}$, where $B^{\prime}={ }^{t}\left(\left(\frac{2}{p_{1}}\right)^{\prime}, \ldots,\left(\frac{2}{p_{t-1}}\right)^{\prime}\right)$, if and only if $A_{E} X_{m}=B$, where $B={ }^{t}\left(\left(\frac{2}{p_{1}}\right)^{\prime}, \ldots,\left(\frac{2}{p_{t}}\right)^{\prime}\right)$, if and only if

$$
\begin{cases}\left(\frac{m}{p}\right)=\left(\frac{2}{p}\right) & \text { for every prime } p \mid d m^{-1}, \\ \left(\frac{d m^{-1}}{p}\right)=\left(\frac{2}{p}\right) & \text { for every prime } p \mid m,\end{cases}
$$

if and only if $2 m \in S_{1}$.

(2) Suppose $d \not \equiv-1 \bmod 4$ and $-1,-2 \notin N F$. Then $D=-4 d$ is the discriminant of $E$ and $p_{t}=2$. For $m \in S_{0}$ and $\varepsilon \in\{1,2\}$, we have $A_{E}^{\prime} X_{\varepsilon m}=$ $B^{\prime}$, where $X_{\varepsilon m}$ is defined as above and $B^{\prime}={ }^{t}\left(\left(\frac{-1}{p_{1}}\right)^{\prime}, \ldots,\left(\frac{-1}{p_{t-1}}\right)^{\prime}\right)$, if and only if

$$
\begin{cases}\left(\frac{\varepsilon m}{p}\right)=\left(\frac{-1}{p}\right) & \text { for every prime } p\left(\neq p_{t}\right) \mid d m^{-1}, \\ \left(\frac{4 d(\varepsilon m)^{-1}}{p}\right)=\left(\frac{-1}{p}\right) & \text { for every prime } p\left(\neq p_{t}\right) \mid m,\end{cases}
$$

if and only if $\varepsilon m \in S_{2}$.

Suppose $d \equiv 3 \bmod 8$ and $-1,-2 \notin N F$. Then $D=-d$ is the discriminant of $E$, odd $m \notin S_{2}$ by the quadratic reciprocity law, and $1=\left(\frac{-2}{d}\right)=$ $\left(\frac{-2}{p_{1}}\right) \ldots\left(\frac{-2}{p_{t}}\right)$. Similarly to (1), we can get the second part of $(2)$.

(3) If $2 \in N F, d=u^{2}-2 w^{2}, u, w \in \mathbb{N}$, and $2(u+w)^{2}=d+(u+2 w)^{2}$, we need only consider the case of $d \equiv-1 \bmod 8$. Let $u+w=2^{i} \overline{u+w}$, where $\overline{u+w}$ is odd. Then

$$
1=\left(\frac{-d}{\overline{u+w}}\right)=\left(\frac{\overline{u+w}}{d}\right)=\left(\frac{u+w}{p_{1}}\right) \ldots\left(\frac{u+w}{p_{t}}\right)
$$

by $2 \in N F, d \equiv-1 \bmod 8$, and the quadratic reciprocity law. Similarly to (1), we can get (3).

(4) It is clear. 
Corollary 3.1. Let $F=\mathbb{Q}(\sqrt{d}), E=\mathbb{Q}(\sqrt{-d}), d>2$ a squarefree integer, -1 or $-2 \in N F$, and $C(E)$ the (narrow) class group of $E$.

(1) If $2 \notin N F$, then the 2-Sylow subgroup of $\mathrm{K}_{2} \mathrm{O}_{F}$ is elementary Abelian if and only if $r_{4}(C(E))=0$.

(2) If $2 \in N F$, then the 2-Sylow subgroup of $\mathrm{K}_{2} \mathrm{O}_{F}$ is elementary Abelian if and only if $r_{4}(C(E))=1$ and the system (3.3) is not solvable, where $B^{\prime}={ }^{t}\left(\left(\frac{u+w}{p_{1}}\right)^{\prime}, \ldots,\left(\frac{u+w}{p_{t-1}}\right)^{\prime}\right)$.

Pr o of. Since -1 or -2 is in $N F, d \not \equiv-1 \bmod 8$ by the quadratic reciprocity law. If $2 \notin N F$, by Theorem 3.1, we can get (1). If $2 \in N F$, then $d \equiv 1$ or $2 \bmod 8$ and $r_{4}(C(E)) \geq 1$, so we can get $(2)$ by Theorem 3.1 .

4. Imaginary quadratic field. For an imaginary quadratic field $E=$ $\mathbb{Q}(\sqrt{-d})$, by [14], we have $\left[\Delta_{E}: E^{* 2}\right]=4$, where $\Delta_{E}=\left\{a \in E^{*} \mid\{-1, a\}=\right.$ $1\}$ is called the Tate kernel of $E$.

J. Browkin and A. Schinzel [2] have given all elements of order 2 of $\mathrm{K}_{2} \mathrm{O}_{\mathrm{E}}$.

Lemma 4.1. Let $E=\mathbb{Q}(\sqrt{-d}), d>2$ a squarefree integer. Then all elements of order at most 2 of $\mathrm{K}_{2} \mathrm{O}_{F}$ are of the form

$$
\left\{-1, m \gamma_{j}\right\}, \quad j=1,2,
$$

where $m$ is an odd positive divisor of $D, \gamma_{1}=1$, and $\gamma_{2}=u+\sqrt{-d},-d=$ $u^{2}-2 w^{2}, u, w \in \mathbb{N}$. Moreover there is a unique $m \gamma_{j}(\neq 1) \in \Delta_{E}$.

In [11], H. Qin has given conditions for $K_{2} O_{E}$ to have elements of order 4.

Lemma 4.2. Let $E=\mathbb{Q}(\sqrt{-d}), F=\mathbb{Q}(\sqrt{d}), d>2$ a squarefree integer, and $m$ an odd positive divisor of $d$.

(1) There is a $\beta \in K_{2} O_{E}$ such that $\beta^{2}=\{-1, m\}$ if and only if there is $\varepsilon \in\{1,2\}$ such that $\varepsilon m \in N F$.

(2) If $2 \in N E,-d=u^{2}-2 w^{2}, u, w \in \mathbb{N}$, then there is a $\beta \in K_{2} O_{E}$ such that $\beta^{2}=\{-1, m(u+\sqrt{-d})\}$ if and only if $m(u+w) \in N F$.

Definition 4.1. Let $E=\mathbb{Q}(\sqrt{-d}), d>2$ a squarefree integer. We define

$$
\begin{aligned}
S_{0} & =\{m \mid m \text { is an odd positive divisor of } d\}, \\
T & =\left\{\varepsilon m \in N F \mid m \in S_{0} \text { and } \varepsilon \in\{1,2\}\right\} .
\end{aligned}
$$

If $2 \in N E,-d=u^{2}-2 w^{2}, u, w \in \mathbb{N}$, we define

$$
T^{\prime}=\left\{m(u+\sqrt{-d}) \mid m \in S_{0} \text { and } m(u+w) \in N F\right\} .
$$

Similarly, $T$ is the group with multiplication $m_{1} \cdot m_{2}=m_{1} m_{2} /\left(m_{1}, m_{2}\right)^{2}$, and $\bar{T}^{\prime}=\left(T^{\prime} \cup T\right)$ is the group generated by the set $T^{\prime} \cup T$ with multiplication $m_{1}(u+\sqrt{-d}) \cdot m_{2}(u+\sqrt{-d})=m_{1} \cdot m_{2}, m_{1} \cdot m_{2}(u+\sqrt{-d})=\left(m_{1} \cdot m_{2}\right)$ $\cdot(u+\sqrt{-d})$. In fact, if $T^{\prime} \neq \emptyset$, then $\bar{T}^{\prime}=(m(u+\sqrt{-d})) \times T$, where 
$m(u+\sqrt{-d}) \in T^{\prime}$. Note that, by [11], there is a $\delta(\neq 1,2) \in T \cup T^{\prime}$ such that $\delta \in \Delta_{E}$.

Lemma 4.3. Notations as above.

(1) If $2 \notin N E$, then $r_{4}\left(K_{2} O_{E}\right)=r_{2}(T)-1$.

(2) If $2 \in N E$, then $r_{4}\left(K_{2} O_{E}\right)=r_{2}(T)+s-2$, where

$$
s= \begin{cases}1 & \text { if } T^{\prime} \neq \emptyset \\ 0 & \text { if } T^{\prime}=\emptyset\end{cases}
$$

Proof. (1) If $2 \notin N E$, then $\alpha: T \rightarrow{ }_{2} K_{2} O_{E} \cap\left(K_{2} O_{E}\right)^{2}, a \mapsto\{-1, a\}$, is a surjective homomorphism and $\operatorname{ker} \alpha=\{1, \varepsilon m\}$, where $\{-1, \varepsilon m\}=1$ and $\varepsilon m \neq 1,2$. Hence $r_{4}\left(K_{2} O_{F}\right)=r_{2}(T)-1$.

(2) If $2 \in N F$, then $\alpha: \bar{T}^{\prime} \rightarrow{ }_{2}\left(K_{2} O_{E}\right) \cup\left(K_{2} O_{E}\right)^{2}, \varepsilon m \gamma_{j} \mapsto\left\{-1, \varepsilon m \gamma_{j}\right\}$, $j=1,2$, is surjective homomorphism and $\operatorname{ker} \alpha=\left\{1,2,2 m \gamma_{j}, m \gamma_{j}\right\}$, where $\left\{-1, m \gamma_{j}\right\}=1$ and $m \gamma_{j} \neq 1,2$. Hence $r_{4}\left(K_{2} O_{E}\right)=r_{2}\left(\bar{T}^{\prime}\right)-2=r_{2}(T)+s-2$, where $s=1$ if $T^{\prime} \neq \emptyset$ or $s=0$ if $T^{\prime}=\emptyset$.

Theorem 4.1. Let $F=\mathbb{Q}(\sqrt{d}), E=\mathbb{Q}(\sqrt{-d}), d>2$ a squarefree integer, and $C(F)$ the narrow class group of $F$.

(1) If $2 \notin N E$, then $r_{4}\left(K_{2} O_{E}\right)=r_{4}(C(F))+s$, where

$$
s= \begin{cases}1 & \text { if } d \equiv 1 \bmod 8 \text { and } 2 m \in T, \\ 0 & \text { otherwise. }\end{cases}
$$

(2) If $2 \in N E$, then

$$
r_{4}\left(K_{2} O_{E}\right)= \begin{cases}r_{4}(C(F))+s^{\prime} & \text { if } d \equiv 1 \bmod 8 \\ r_{4}(C(F))+s^{\prime}-1 & \text { if } d \neq 1 \bmod 8\end{cases}
$$

where

$$
s^{\prime}= \begin{cases}1 & \text { if } T^{\prime} \neq \emptyset \\ 0 & \text { if } T^{\prime}=\emptyset\end{cases}
$$

Moreover, $r_{4}\left(K_{2} O_{E}\right)=r_{4}(C(F))+a(E)$, where $a(E)=-1$, 0 , or 1 is determined by $E$.

Proof. By Lemma 4.3, the relation between $T$ and $D(N F)$, and by $r_{4}(C(F))=r_{2}(D(N F))-1$, we get the result.

Corollary 4.1. Notations as above.

(1) If $2 \notin N E$, then $r_{4}\left(K_{2} O_{E}\right)=0$ if and only if $r_{4}(C(F))=0$, and $2 m \notin T$ if $d \equiv 1 \bmod 8$.

(2) If $2 \in N E$ and $d \equiv 1 \bmod 8$, then $r_{4}\left(K_{2} O_{E}\right)=0$ if and only if $r_{4}(C(F))=0$ and $T^{\prime}=\emptyset$.

(3) If $2 \in N E$ and $d \not \equiv 1 \bmod 8$, then $r_{4}\left(K_{2} O_{F}\right)=0$ if and only if $r_{4}(C(F))=1$ and $T^{\prime}=\emptyset$, or $r_{4}(C(F))=0$ and $T^{\prime} \neq \emptyset$. 
TheOREM 4.2. Let $F=\mathbb{Q}(\sqrt{d}), E=\mathbb{Q}(\sqrt{-d}), d>2$ a squarefree integer, $C(F)$ the narrow class group of $F$, and $A_{F}^{\prime}$ defined as in (2.2).

(1) If $2 \notin N E$ and $d \equiv 1 \bmod 8$, then there is even $2 m \in T$ if and only if the system of equations

$$
A_{F}^{\prime} X=B^{\prime}
$$

is solvable, where $A_{F}^{\prime}$ is defined as in $(2.2)$ and $B^{\prime}={ }^{t}\left(\left(\frac{2}{p_{1}}\right)^{\prime}, \ldots,\left(\frac{2}{p_{t-1}}\right)^{\prime}\right)$.

(2) If $2 \in N F,-d=u^{2}-2 w^{2}, u, w \in \mathbb{N}$, then $T^{\prime} \neq \emptyset$ if and only if the system (4.1) is solvable, where $B^{\prime}={ }^{t}\left(\left(\frac{u+w}{p_{1}}\right)^{\prime}, \ldots,\left(\frac{u+w}{p_{t-1}}\right)^{\prime}\right)$.

Pr o of. Proceed as in the proof of Theorem 3.2.

Let $F=\mathbb{Q}(\sqrt{d})$ be a real quadratic field. By genus theory, there is a unique $q(\neq 1) \in D(N F)$ such that $Q^{2}=(q)$ and $\operatorname{cl}(Q)=1$ in the narrow class group $C(F)$. We call the $q$ the dependent divisor of ambiguous ideals of $F$. Suppose $r_{4}\left(K_{2} O_{E}\right)=0$. We set up a relation between the Tate kernel of $\mathrm{K}_{2} \mathrm{O}_{\mathrm{E}}$ and the dependent divisor of ambiguous ideals of $F$.

Theorem 4.3. Let $F=\mathbb{Q}(\sqrt{d}), E=\mathbb{Q}(\sqrt{-d}), d>2$ a squarefree integer. Suppose $r_{4}\left(K_{2} O_{E}\right)=0$. Then, if $q(\neq 2)$ is the dependent divisor of ambiguous ideals of $F, \Delta_{E}=(\{2, q\}) E^{* 2}$; if 2 is the dependent divisor of ambiguous ideals of $F, \Delta_{E}=(\{2, m(u+\sqrt{-d})\}) E^{* 2}$, where $m(u+\sqrt{-d}) \in T^{\prime}$.

Pro of. If $2 \notin N E$ and $r_{4}\left(K_{2} O_{E}\right)=0$, then, by Corollary 4.1, $r_{4}(C(F))$ $=0$, and $2 m \notin T$ if $d \equiv 1 \bmod 8$. Hence $\operatorname{rank} A_{F}=t-1$ and there is a unique $q(\neq 1,2) \in D(N F)=T$ such that $A_{F} X_{q}=0$. Therefore $q$ is the dependent divisor of ambiguous ideals of $F$ and $q \in \Delta_{E}$.

If $2 \in N E, d \equiv 1 \bmod 8$ and $r_{4}\left(K_{2} O_{E}\right)=0$, then by Corollary 4.1 , we have the same result as above.

If $2 \in N E, d \not \equiv 1 \bmod 8$ and $r_{4}\left(K_{2} O_{E}\right)=0$, then by Corollary 4.1, we need to consider two cases.

The first case: $r_{4}(C(F))=1$ and $T^{\prime}=\emptyset$. Then $\operatorname{rank} A_{F}=t-2$ and $\bar{T}^{\prime}=T=D(N F)$. Hence $D(N F)=\{1,2, q, 2 q\}$. Suppose that 2 is the dependent divisor of ambiguous ideals of $F$. Since $2(u+w)^{2}=(u+2 w)^{2}-d$, we have $((u+2 w)+\sqrt{d})=Q_{2} Q_{u+w}^{2}$, where $Q_{2}$ and $Q_{u+w}$ are ideals of $F$ with $Q_{2}^{2}=(2)$ and $Q_{u+w} Q_{u+w}^{\prime}=(u+w)$. Then $\operatorname{cl}\left(Q_{u+w} 2\right)^{2}=\operatorname{cl}\left(Q_{2}\right)=1$. Hence, by genus theory, $\operatorname{cl}\left(Q_{u+w}\right)=\operatorname{cl}\left(Q_{m}\right)$, where $Q_{m}$ is an ideal of $F$ with $Q_{m}^{2}=(m)$ and $m \in D(F)$. So $\operatorname{cl}\left(Q_{u+w} Q_{m}\right)=1 \in C(F)^{2}$ and $m(u+w)=N_{F / \mathbb{Q}}\left(Q_{u+w} Q_{m}\right) \in N F$, contrary to $T^{\prime}=\emptyset$. Therefore, $q$ or $2 q$ is the dependent divisor of ambiguous ideals of $F$ and $q, 2 q \in \Delta_{E}$.

The second case: $r_{4}(C(F))=0$ and $T^{\prime} \neq \emptyset$. Then $\operatorname{rank} A_{F}=t-1$, $D(N F)=T=\{1,2\}$, and $\bar{T}^{\prime}=(m(u+\sqrt{-d})) \times T$. Hence 2 is the dependent divisor of ambiguous ideals of $F$ and $m(u+\sqrt{-d}) \in \Delta_{E}$. 
Question. Suppose $r_{4}\left(K_{2} O_{E}\right) \geq 1$. Do we have results similar to Theorem 4.3 ?

Acknowledgements. The authors would like to thank the referee for helpful suggestions which resulted in the present version.

\section{References}

[1] B. Brauckmann, The 2-Sylow-subgroup of the tame kernel of number fields, Canad. J. Math. 43 (1991), 255-264.

[2] J. Browkin and A. Schinzel, On Sylow 2-subgroups of $\mathrm{K}_{2} \mathrm{O}_{F}$ for quadratic fields $F$, J. Reine Angew. Math. 331 (1982), 104-113.

[3] A. Candiotti and K. Kramer, On the 2-Sylow subgroup of the Hilbert kernel of $K_{2}$ of number fields, Acta Arith. 52 (1989), 49-65.

[4] P. E. Conner and J. Hurrelbrink, The 4-rank of $\mathrm{K}_{2} \mathrm{O}_{F}$, Canad. J. Math. 41 (1989), 932-960.

[5] K. Q. Feng, Non-congruent numbers, odd graphs and the Birch-Swinnerton-Dyer conjecture, Acta Arith. 75 (1996), 71-83.

[6] E. Hecke, Lecture on the Theory of Algebraic Numbers, Grad. Texts in Math. 77, Springer, 1981.

[7] J. Hurrelbrink, Circular graphs and 4-ranks of ideal class groups, Canad. J. Math. 46 (1994), 169-183.

[8] J. Hurrelbrink and M. Kolster, Tame kernels under relative quadratic extensions and Hilbert symbols, J. Reine Angew. Math. 499 (1998), 145-188.

[9] M. Kolster, The structure of the 2-Sylow subgroup of $K_{2}(O), I$, Comment. Math. Helv. 61 (1986), 376-388.

[10] H. Qin, The 2-Sylow subgroups of the tame kernel of imaginary quadratic fields, Acta Arith. 69 (1995), 153-169.

[11] —, The 4-rank of $\mathrm{K}_{2} \mathrm{O}_{F}$ for real quadratic fields $F$, ibid. 72 (1995), 323-333.

[12] L. Rédei, Arithmetischer Beweis des Satzes über die Anzahl der durch 4 teilbaren Invarianten der absoluten Klassengruppe im quadratischen Zahlkörper, J. Reine Angew. Math. 171 (1935), 55-60.

[13] Y. Sueyoshi, On a comparison of the 4-ranks of the narrow ideal class groups of $\mathbb{Q}(\sqrt{d})$ and $\mathbb{Q}(\sqrt{-d})$, Kyushu J. Math. 51 (1997), 261-272.

[14] J. Tate, Relations between $K_{2}$ and Galois cohomology, Invent. Math. 36 (1976), $257-274$.

Institute of Mathematics

Fudan University

Shanghai 200433, P.R. China

Department of Mathematics

Xuzhou Normal University

Jiangsu 221009, P.R. China

E-mail: yue-qin@263.net
Department of Mathematics Tsinghua University Beijing 100000, P.R. China E-mail: kqfeng@public.bta.net.cn

Received on 19.1.1999

and in revised form 1.12.1999 